\title{
Accelerating Public Health Situational Awareness through Health Information Exchanges: An Annotated Bibliography
}

\author{
Debra Revere ${ }^{1}$, Kevin C. Stevens ${ }^{2}$ \\ ${ }^{1}$ Center for Public Health Informatics, University of Washington, Seattle, WA \\ ${ }^{2}$ Marion County Health Department, Indiana
}

\begin{abstract}
In 2008, the Centers for Disease Control and Prevention awarded contracts to health information exchanges in Indiana, New York and Washington/Idaho to accelerate public health situational awareness. Awardees in each state have disseminated their findings and lessons at professional conferences and in peer-reviewed journals. The dissemination formats ranged from papers, oral presentations, posters, panels and demonstrations at interoperability showcases. With a focus on health information exchange and public health, topics included biosurveillance, electronic laboratory reporting, broadcast messaging, and notifiable disease surveillance. Each presentation is summarized in this bibliography, and the authors affiliated with each site are highlighted.
\end{abstract}

Keywords: biosurveillance, situational awareness, electronic laboratory reporting, health information exchange, notifiable diseases

\section{Introduction}

With funding from the Centers for Disease Control and Prevention (CDC) in February 2008, the "Accelerating Public Health Situational Awareness through Health Information Exchanges" project was initiated in three locations: Indiana, New York and Washington/Idaho. This paper is an annotated bibliography of the peer-reviewed and grey literature publications and presentations by the three Health Information Exchanges (HIEs). These HIEs implemented the capability to electronically move clinical information between disparate health care and public health information systems while maintaining the meaning of the information being exchanged. For this project, HIEs have developed real-time, nationwide public health event-monitoring capability to assist with and improve early event detection, public health situational awareness, outbreak management, and countermeasure and response administration. In addition, the HIEs are collaborating on a consortia level to cooperatively develop specifications for and trial implementations of the emerging and interoperable "network of networks" that is the Nationwide Health Information Network (NHIN).

Each HIE represents a wealth of innovative patient- and population-based experience from public health practice, academia, business, and computing and information technology fields. Furthermore, each HIE has contributed to improving the operation of information technology systems and secure exchange of data and information to enhance early detection and a rapid 
response to and management of public health emergencies. This annotated bibliography represents the material outputs of each HIE's participation in the accelerating public health situation awareness initiative funded by the CDC.

\section{Acronyms Used:}

AHIC American Health Information Community

CSTE Council for State and Territorial Epidemiologists

EHR Electronic Health Record

ELR Electronic Laboratory Reporting

EMR Electronic Medical Record

HIE Health Information Exchange

HIMSS Health Information Management and Systems Society

HITSP Healthcare Information Technology Standards Panel

IHE Integrating the Healthcare Enterprise

MBDS Minimum Biosurveillance Data Set

NEDSS National Electronic Disease Surveillance System

NHIN Nationwide Health Information Network

ONC Office of the National Coordinator for Health Information Technology

PHIN Public Health Information Network

RHIO Regional Health Information Organization

\section{Presentations}

Summaries of the presentations by awardees of the CDC contract are listed in descending chronological order. Authors affiliated with each site are highlighted in bold.

\section{0}

Stevens KC, Grannis S, Gibson PJ, Merriwether R. Local public health and health information exchange business model. AMIA NOW! May 2010.

Summary: This poster describes the interaction between local public health departments and health information exchanges in the development of a business model. The business model incorporates the Indiana Health Information Exchange services: clinical repository (reportable laboratory results), clinical messaging (public health alerts) and clinical quality services (chronic disease management). http://amianow2010.amia.org/files/2010-AMIA-NOW-Final-Program.pdf (p. 26)

Stevens KC. The role of a public health informatician at a health information exchange. AMIA NOW! May 2010.

Summary: This poster describes how a public health informatician (PHI) at a HIE identifies and evaluates the quality and quantity of clinical data that is sent to public health. The role of a PHI at a HIE is compared to the CDC "Competencies for Public Health Informatician" and future implications for public health in the context of the NHIN.

http://amianow2010.amia.org/files/2010-AMIA-NOW-Final-Program.pdf (p. 26) 
Karras B, Gibson J, Johnson G. Public health and health information exchange: Lessons learned - a Centers for Disease Control and Prevention sponsored initiative in New York, Indiana and Washington/Idaho. 2010 CSTE Annual Conference, June 2010.

Summary: A presentation demonstrating how collaborative efforts between public health and HIE to enable electronic information exchange between clinical and public health settings can improve and transform public health capabilities. Representatives from the three regions presented lessons learned and the organizational/technological infrastructure from the work that has been done to date.

http://www.cste.org/conference/AgendaandEvents/tabid/383/Default.aspx

Painter I, Bugni P, Trebatoski M, Revere D, Karras B, Dobbs D, Lober W. Potential to estimate vaccine uptake in an emerging epidemic using data from a Health Information Exchange. 2010 CSTE Annual Conference, June 2010.

Summary: A report regarding the ability of HIEs to provide real-or near real-time vaccination information during an epidemic when obtaining vaccination uptake information is critical for decision-making about targeted vaccination efforts. Using data obtained through the Northwest Public Health Information Exchange (NWPHIE), the authors reported that sufficient selfreported vaccination data is present in the HIE data to make it usable for vaccination surveillance. In addition, in comparison to other methods for obtaining this data, NWPHIE provides nearly real-time provision of relevant data for surveillance by Washington State Department of Health.

http://www.cste.org/conference/AgendaandEvents/tabid/383/Default.aspx

Karras B. Public Health: Case Reporting, Biosurveillance and Immunization Tracking. HIMSS10 Annual Conference Interoperability Showcase, March 2010.

Summary: This presentation describes the use of HITSP standards and IHE profiles to support public health functions such as biosurveillance, case reporting and immunization tracking. Also discussed are the current practices and an overview of the demonstrations taking place in a model HIE represented at the HIMSS Interoperability Showcase.

http://himssconference.org/education/sessiondetail.aspx?eventID=4116

Dixon B. Bi-Directional Communication: Enhancing Situational Awareness in Public Health and Clinical Practice. HIMSS10 Annual Conference Interoperability Showcase, March 2010.

Summary: This presentation described how a health information exchange could facilitate efficient and effective dissemination of information to clinicians to keep them informed of emerging public health threats. A new component of the Indiana Network for Patient Care that delivers information in real-time from local public health agencies and integrated into existing clinical workflow was described, and a real-world example from the 2009 H1N1 outbreak was demonstrated.

http://himssconference.org/education/sessiondetail.aspx?eventID=4124

\section{9}

Magruder C, Johnson G, Grannis S, Karras B. Enhancing Public Health Practice Through Health Information Exchange - New York, Indiana, and Washington/Idaho Perspectives. 2009 CSTE Annual Conference, June 2009. 
Summary: This panel presentation demonstrates how organizational and technological infrastructure developed for clinical data exchange can enhance public health activities. An interoperable network of systems to share information among medical providers, HIEs, and public health at the local, state, and federal levels is being implemented through innovative technologies and development of business, policy, and governance rules.

http://www.cste.org/dnn/AnnualConference/2009AnnualConferenceArchive/tabid/321/Default.a $\underline{\operatorname{spx}}$

Safran C, Garrett N, Dente M, Le L, Grannis S, Karras B. Panel: Translating Public Health Information into Clinical Action: A National Demonstration Project / Connecting Public Health to Clinical Care through Health Information Exchange - Implementation of AHIC Biosurveillance. AMIA Spring Congress, May 2009.

Summary: Panel presentation of two projects. The first presentation reported on a publicprivate partnership that is extending the capability of public health agencies to communicate with EMR systems, using a standard messaging format and other data standards (e.g. HITSP) to create actionable and consumable alerts for providers. The second presentation reported on the HIE collaboration for improving public health situational awareness through establishing and enhancing public health services in existing HIEs.

http://2009springcongress.amia.org/files/congress2009/AMIA_Spring_Congress_On-

Site_Program.pdf

Dixon BE, Grannis S. Enhancing public health surveillance and communication through health information exchange. AMIA Spring Congress, May 2009.

Summary: The authors present recent technologies developed to improve detection and reporting of disease outbreaks, including ELR that enhances public health monitoring, communication, and workflow. Also presented are mechanisms for sending MBDS data to public health in real-time, and how public health can increase the timeliness of information it disseminates to clinicians.

http://2009springcongress.amia.org/files/congress2009/AMIA_Spring_Congress_On-

Site_Program.pdf (Information Sharing Presentations, S27)

Shepherd D, Friedlin J, Grannis S, Hui S, Kho A. A comparison of automated methicillinresistant Staphylococcus aureus identification with current infection control practice. AMIA Annu Symp Proc; 2009:594-8.

Summary: Using data from infection control providers (ICPs), the microbiology lab, and a Regional Healthcare Information Exchange, the authors compared the accuracy of automated identification of MRSA using HL7 lab result messages to current manual infection control practices at a local hospital during July-September 2008. The study concluded that an automated processing of HL7 lab report messages is a more sensitive method of capturing MRSA cases than current standard infection control practice, with minimal loss of specificity.

PubMed Abstract: http://www.ncbi.nlm.nih.gov/pubmed/20351924

Free full text: http://www.ncbi.nlm.nih.gov/pmc/articles/PMC2815488/?tool=pubmed

Grannis SJ, Dixon BE, Gibson J, Smiley A, Dearth S, Stevens K, Barnes M. Design and deployment of an integrated, standards-based public health alerting system. 2009 PHIN Annual Conference, August 2009. 
Summary: A presentation of the system deployed by the Marion County Health Department to deliver public-health alerts to directly to the clinical inbox of clinicians. The system was first used during the HIN1 influenza outbreak. In addition to broadcasting to all physicians within the system the system is capable of targeting customized combinations of physician specialties and geographic regions.

Abstract: http://cdc.confex.com/cdc/phin2009/webprogram/Paper21186.html

Hills RA, Lober WB, Dixon B, Grannis S, Lombardo J, Revere D. Demonstrating Interoperability: Biosurveillance in a Model Health Information Exchange. 2009 PHIN Annual Conference, August 2009.

Summary: A summary of the HIMSS IHE demonstration that explored the feasibility of enhancing biosurveillance using interoperability specifications within a model HIE. The scenario focused on a Salmonella outbreak and demonstrated the IHE infrastructure that enhanced communication between public health agencies as they monitored the outbreak and initiated alerts to providers. The project successfully illustrated the feasibility of utilizing an HIE infrastructure for outbreak detection, alerting, and other public health functions.

http://cdc.confex.com/cdc/phin2009/webprogram/Paper21087.html

Hills RA, Lober WB, Baseman JG, Sibley J. Xforms for Public Health: Notifiable Condition Case Reporting and Public Health Alerting Using IHE's RFD Profile. 2009 PHIN Annual Conference, August 2009.

Summary: Public health alerting and notifable condition reporting rely on bi-directional communication. This presentation reviewed the HIMSS IHE 2009 demonstration that successfully used of Retrieve Forms for Data Capture (RFD) and its underlying Xforms standard for two public health use-cases.

http://cdc.confex.com/cdc/phin2009/webprogram/Paper20771.html

Lober WB, Magruder C, Grannis S, Soulakis N. Sharing a Summarized Public Health Surveillance Data Set (SPHSDS). 2009 PHIN Annual Conference, August 2009.

Summary: Presentation of a model for using a summarized public health surveillance data set (SPHSDS) to support public health situational awareness that delivers summarized biosurveillance data before more detailed patient-level data is revealed when public health events of interest are identified. Various approaches for using a SPHSDS are discussed, including plans for using a SPHSDS by the CDC's three "Situational Awareness through HIE" awardees in NY, IN and WA State / Idaho.

http://cdc.confex.com/cdc/phin2009/webprogram/Paper21110.html

Lober WB, Hills R, Revere D, Kirnack A. Towards a Unified Framework of Public Health Knowledge for Clinical Decision Support. 2009 PHIN Annual Conference, August 2009.

Summary: While alerting is one of the traditional responsibilities of public health agencies, real-time computerized decision support; those developing clinical systems have, primarily addressed including immunization decision support. This presentation covered commonalities between the HIMSS IHE 2009 alerting and immunization scenarios and highlighted the way standards supported tailored decision support in both cases. The authors suggest that these commonalities represent a unified approach to knowledge management and retrieval.

http://cdc.confex.com/cdc/phin2009/webprogram/Paper21165.html 
Magruder C, Dobbs D, Karras B, Blake PA, Grannis S, Johnson GS. Public Health and Health Information Exchanges: Developing a Common Roadmap to Future Success. 2009 PHIN Annual Conference, 2009.

Summary: A presentation that examined the benefits of and best practices for sharing health information electronically between HIEs and public health. In particular, the following issues are highlighted: collaboration and data sharing agreements; NHIN standards and Gateway services; innovative information-sharing practices; extending existing technical standards to support summarized data exchange; rResponding to the HIN1 outbreak using HIE data and processes; and evaluation plans for assessing the utility and effectiveness of HIE and public health interactions.

http://cdc.confex.com/cdc/phin2009/webprogram/Paper21174.html

Johnson GS, Wu W, Davissson M, Grannis S, Abellera J, Magruder C. Health Information Exchange and Notifiable Disease Surveillance. 2009 PHIN Annual Conference, August 2009.

Summary: Review of the approach to assessing the utility of HIEs to support surveillance and notifiable disease case reporting as focused on acute hepatitis B virus (HBV) infection. Assessments will build upon established exchange of the AHIC MBDS between HIEs and their respective public health agencies. Approaches to evaluating the MBDS in the context of notifiable disease case reporting, identifying and assessing the availability of additional data elements necessary to conduct acute $H B V$ surveillance, comparing results with existing surveillance mechanisms, and assessing the impact in public health jurisdictions with and without existing ELR mechanisms is covered.

http://cdc.confex.com/cdc/phin2009/webprogram/Paper21148.html

Grannis S, Friedlin J, Overhage JM, Merriwether R. Practical Aspects of an Operational Open Source Notifiable Condition Detection System. 2009 PHIN Annual Conference, August 2009.

Summary: Building on standards for message structure and content (HL7 and LOINC ${ }^{\circledR}$ ), the Indiana Network for Patient Care HIE has implemented and maintained an automated notifiable condition reporting system that receives real-time HL7 clinical results from a variety of HIE stakeholders, translates these disparate proprietary codes into LOINC codes, determines whether the results carried by the message indicates a notifiable condition by checking the abnormal flag sometimes contained in the message, or by comparing the test results with criteria in the PHIN notifiable conditions mapping table. This presentation covered expansion of the notifiable condition processor infrastructure to create a modular notifiable condition processor, the--Notifiable Condition Detector (NCD)--which is re-usable by other HIEs and public health stakeholders.

http://cdc.confex.com/cdc/phin2009/webprogram/Paper21179.html

\section{8}

Doctor JN, Baseman JG, Lober WB, Davies J, Kobayashi J, Karras BT, Fuller S. Timetradeoff utilities for identifying and evaluating a minimum data set for time-critical biosurveillance. Med Decis Making 2008; 28(3):351-8. Epub 2008 May 13.

Summary: The authors establish and evaluate a method for measuring the utility of biosurveillance data using Multi-Attribute Utility Theory (MAUT). MAUT is used to assess and 
rank the risks of a decision-maker's preferences. In this paper, the authors apply this theory to measure the utility of biosurveillance data using a time-tradeoff exercise. Empirically, the method shows initial promise for evaluating a minimum data set for biosurveillance. The authors suggest that future applications of this approach may prove useful in disease surveillance planning and evaluation.

PubMed Abstract: http://www.ncbi.nlm.nih.gov/pubmed/18480039

Gotham IJ, Le LH, Sottolano DL, Schmit KJ. Public Health Preparedness Informatics Infrastructure. A Case Study in Integrated Surveillance and Response: 2004-2005 National Influenza Vaccine Shortage. Lecture Notes in Bioinformatics, vol 5354 (Proceedings of the 2008 International Workshop on Biosurveillance and Biosecurity). Berlin: Springer, pp. 42-55.

Summary: Integrating information systems within an informatics framework supporting a community of information trading partners engaged in routine HIE can support key public health emergency preparedness (PHEP) activities. The authors describe how this framework supported and enhanced the efficacy of New York's response to the announcement of significant influenza vaccine shortfalls in 2004. This PHEP event required a full array of integrated and heightened activities at state and local levels. The authors describe how the presence of the HIE framework supported advanced preparedness and just-in-time response, and detail related performance metrics and lessons learned from the response.

Abstract: http://portal.acm.org/citation.cfm?id=1485119.1485125

Hills RA, Lober WB, Painter IS. Biosurveillance, Case Reporting, and Decision Support: Public Health Interactions with a Health Information Exchange. Lecture Notes in Bioinformatics, vol 5354 (Proceedings of the 2008 International Workshop on Biosurveillance and Biosecurity). Berlin: Springer, pp. 10-21.

Summary: This paper describes support for three public health practice domainsbiosurveillance, case reporting, and communication from public health to providers through integrated decision support-in demonstrations of a model HIE. The model HIE implements interoperability through the use Integration Profiles standards that support specific data transfer use cases. These methods were validated for each public health domain in a distributed environment in national showcase demonstrations. The authors believe that this work has implications for the integration of public health functions into any HIE, regardless of architecture and may be extended to strengthen development of the Public Health Grid.

Abstract: http://www.springerlink.com/content/k4701h773010v367/

Overhage JM, Grannis S, McDonald C. A comparison of the completeness and timeliness of automated ELR and spontaneous reporting of notifiable conditions. Am J Public Health 2008; 98(2): 344-350.

Summary: In a comparison of traditional spontaneous paper-based reporting to automated ELR through the HIE, the authors found that automated ELR of notifiable-diseases results in information being delivered to public health departments more completely and quickly than is the case with spontaneous, paper-based reporting.

PubMed Abstract: http://www.ncbi.nlm.nih.gov/pubmed/18172157

Free full text at http://www.ncbi.nlm.nih.gov/pmc/articles/PMC2376898/ 
Painter I, Hills RA, Lober WB, Randels KM, Sibley J, Webster E. Extending Functionality of and Demonstrating Integrated Surveillance for Public Health within a Prototype Regional Health Information Exchange. AMIA Annu Symp Proc. 2008:969.

Summary: A poster describing the HIMSS 2008 conference demonstration of how multijurisdictional public health surveillance and monitoring processes could be supported and expedited through integration with a prototype HIE.

PubMed Abstract: http://www.ncbi.nlm.nih.gov/pubmed/18999244

Friedlin J, Grannis S, Overhage JM. Using Natural Language Processing to improve accuracy of automated notifiable disease reporting. AMIA Annu Symp Proc. 2008:207-11.

Summary: The authors report on the results of applying a natural language processing (NLP) system to automated ELR, focusing on methicillin-resistant Staphylococcus aureus (MRSA). The NLP system improved accuracy and completeness for MRSA, and achieved high sensitivity, specificity, positive predicted value and F-measure. The authors suggest that using NLP can improve the completeness and accuracy of automated ELR.

PubMed Abstract: http://www.ncbi.nlm.nih.gov/pubmed/18999177

Free full text at http://www.ncbi.nlm.nih.gov/pmc/articles/PMC2656046/

Magruder C, Brady J, Dobbs D, Grannis S, Le LH. Enhancing Public Health Capabilities Through Health Information Exchanges - A New Five-Year Initiative in New York, Indiana and Washington/Idaho. 2008 PHIN Annual Conference, August 2008.

Summary: A presentation by each HIE of the individual and collaborative vision of enabling bidirectional sharing of clinically relevant information to improve situational awareness and case reporting. The NIHN backbone for information sharing is described. Also covered are plans for completing an evaluation of the Minimum Dataset to assess its use for responding to and managing high-risk morbidity and mortality events such as seasonal or pandemic influenza, and its adherence to the HIPAA minimum necessary requirement.

http://cdc.confex.com/cdc/phin2008/webprogram/Session9221.html

Grannis S, Biondich P, Downs S, Shelley M, Anand V, Egg J. Leveraging open-source matching tools and Health Information Exchange to improve newborn screening follow-up. 2008 PHIN Annual Conference, August 2008.

Summary: The value of sharing data among HIEs and public health is demonstrated by improving newborn screening follow-up by identifying infants who may lack screening. This use case requires a robust matching process for linking vital records data with newborn screening results to identify unscreened infants. The Indiana Network for Patient Care has implemented a configurable, generalized probabilistic method to perform the matching using an open-source probabilistic matching tool. The design and implementation of the system is described and results for matching infant data across multiple organizations is presented. http://cdc.confex.com/cdc/phin2008/webprogram/Paper17722.html

Grannis S, Overhage JM, Friedlin J. Architectural and operational components of a real world operational automated notifiable condition processor. 2008 PHIN Annual Conference, August 2008.

Summary: Presentation of the Indiana Network for Patient Care, an operational HIE built on standards for message structure and content (HL7 and LOINC $\left.{ }^{\circledR}\right)$, that has proven reliable in 
delivering results and has scaled to multiple clinical data sources over several years of use. The presentation covered implementation of a notifiable condition processor in the context of an HIE rather than at local hospitals, including the technical architecture, and operational aspects of the system.

http://cdc.confex.com/cdc/phin2008/webprogram/Paper17733.html

Le LH, Gotham IJ, Evans LM, Mostashari F, Morse D, Soulakis N, Fuhrman J, Brady J, Ciampa M. Universal Public Health Node: An Evolution of Health Information Exchange for Public Health in New York. 2008 PHIN Annual Conference, August 2008.

Summary: A presentation of the vision and architecture of the Universal Public Health Node (UPHN). The example of New York State is used to demonstrate how the UPHN will address nationwide situational awareness by connecting public health in the HIE with a clinical-based RHIO partners. This connection is accomplished through a common set of standards and services for the bi-directional exchange of data while ensuring appropriate privacy protection. Also presented were four novel functionalities of the UPHN that support multiple public health scenarios and are needed to implement and extend the Biosurveillance Use Case and the Minimum Dataset.

Abstract: http://cdc.confex.com/cdc/phin2008/webprogram/Paper17377.html

Tokars J, Karras BT, Dobbs D, Blake P, Stephens CM, Fraser J, Howell JF, Stevens KC, Dixon BE, Morse D, Le LH, Magruder C. Biosurveillance: Coordinating a Nationwide Approach. $5^{\text {th }}$ Nationwide Health Information Network Forum, December 2008

Summary: Presentation demonstrating how the AHIC Biosurveillance Use Case can be implemented within the NHIN. The presentation further describes the roles that the CDC Biosense program, HIEs, and state and local health departments are playing in the situational awareness contracts funded by the $C D C$.

http://healthit.hhs.gov/portal/server.pt?open=18\&objID=849959\&parentname=CommunityPage

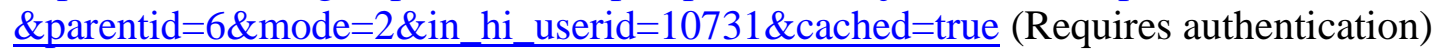

Turner AM, Stavri Z, Revere D, Altamore R. From the ground up: determining the information needs and resources of public health nurses in an Oregon County Health Department. J Med Libr Assoc 2008; 96(4): 335-42.

Summary: The authors identified and assessed information needs and resources of public health nurses in a local health department using the qualitative approach of constant comparative method on semi-structured in-depth interviews with seventeen public health nurses at a local health department in rural Oregon. Major themes that emerged included: (1) differences in information needs depending on position and role; (2) colleagues as the most efficient and trusted source of information; (3) limitations of existing knowledge-based resources; (4) need for up-to-date and pertinent information; and (5) need for personal computers, basic communications software, and expanded Internet access.

Free full text: http://www.ncbi.nlm.nih.gov/pmc/articles/PMC2568844/

PubMed Abstract: http://www.ncbi.nlm.nih.gov/pubmed/18974810

\section{Conflicts of interest}

The authors have no conflicts of interest to report at this time. 


\section{Acknowledgement}

The authors would like to thank Brian Dixon for his comments on this manuscript. This project was funded by the Centers for Disease Control \& Prevention under contract 200-2008-24368.

\section{Correspondence}

Debra Revere: drevere@u.washington.edu 